\title{
Adiabatic compression of a trapped Fermi gas
}

\author{
L. Viverit ${ }^{1}$, S. Giorgini ${ }^{2}$, L.P. Pitaevskii ${ }^{2,3}$ and S. Stringari ${ }^{2}$ \\ ${ }^{1}$ Ørsted Laboratory, H.C. Ørsted Institute, Universitetsparken 5, DK-2100 Copenhagen Ø, Denmark \\ ${ }^{2}$ Dipartimento di Fisica, Università di Trento, \\ and Istituto Nazionale di Fisica della Materia, I-38050 Povo, Italy \\ ${ }^{3}$ Kapitza Institute for Physical Problems, 117454 Moscow, Russia
}

We propose a method to reach conditions of high degeneracy in a trapped Fermi gas, based on the adiabatic transfer of atoms from a magnetic to a tighter optical trap. The transformation yields a large increase of the Fermi energy, without a significant change of the temperature. The large enhancement of the central density emphasizes the role of the interactions and makes the system much closer to the BCS transition. An estimate of the time needed to achieve the conditions of adiabaticity is also given.

The experimental realization of a highly degenerate atomic Fermi gas confined in traps is a task of primary importance, especially in view of the perspective of approaching the BCS transition to the superfluid phase. The regime of quantum degeneracy has been already reached in a sample of potassium atoms [1], where first signatures of Fermi statistics, like the deviation of the velocity distribution from a Boltzmann profile and the increase of the kinetic energy with respect to the classical value, have been observed. The main difficulties in further lowering the temperature are due to the fact that the efficiency of the evaporative cooling process is strongly quenched [2,3]. In fact Fermi statistics inhibits collisional processes at low temperature both directly, by reducing the phase space available for collisions, and indirectly by lowering the density of the sample because of Pauli repulsion. Procedures to optimize the evaporative process have recently permitted to reach lower temperatures, of the order of $0.2 \sim 0.3 T_{F}$ where $T_{F}$ is the Fermi temperature [2].

The purpose of this work is to propose a method to reach conditions of high degeneracy, based on an adiabatic compression of the gas. A similar method has proven quite successful in producing Bose-Einstein condensation in a reversible way, starting from a trapped Bose gas above the critical temperature [4]. The main point is that, by changing the shape of the confinement from a harmonic to a non harmonic trap, one can increase the degree of quantum degeneracy by keeping the entropy of the total system constant [5]. At the same time, the process of thermalization is not drastically quenched and in typical experimental conditions takes place over times much shorter than the lifetime of the cloud. In the following we consider a gas occupying two different spin states, initially confined by a harmonic trap. We then switch on adiabatically a second tighter trap (see Fig. 1). Experimentally this can be realized using a magnetic trap for the first confinement and an optical trap for the second one. As a consequence of the adiabatic process a fraction of atoms will move from the magnetic to the optical trap. This can provide several important advantages:

i) The gas in the optical trap becomes much more degenerate than the original one. In particular, if the number of atoms transferred to the optical trap is a small fraction, the temperature will not change significantly with respect to the initial value, but the Fermi energy will increase, in a way proportional to the depth of the optical trap.

ii) The gas in the optical trap is much denser due to the tighter confinement. This produces an increase of interaction effects and hence of the value of the critical temperature. Both effects i) and ii) favour the reachability of the BCS transition.

iii) The Fermi energies of the two spin components, which initially were different because of the different magnetic trapping, become closer in the optical trap, thereby favouring the mechanism of Cooper pairing.

Another important advantage of the proposed method is that the velocity distributions of the atomic clouds occupying the magnetic and optical traps can be measured separately. By releasing first the magnetic trap, one can measure the temperature of the sample. The effects of quantum degeneracy can then be investigated by measuring the velocity distribution of the gas confined in the optical trap.

In the first part of the work we assume that thermodynamic equilibrium is ensured during each step of the adiabatic compression and we explore the properties of the new gas produced in the tight confinement by imposing entropy conservation. In the second part of the work we provide estimates for the times required to ensure adiabaticity and discuss possible scenarios where faster adiabatic trasformations take place out of thermal equilibrium.

Let us consider a gas initially confined in a harmonic trap (hereafter called magnetic trap). We assume that the trapping frequencies and the number of atoms are the same for the two spin species. The Fermi energy is given by $\epsilon_{F}^{0}=\hbar \omega_{m a g}(6 N)^{1 / 3}$, where $N$ is the number of atoms of each species and $\omega_{\text {mag }}$ is the geometrical average of the frequencies characterizing the magnetic trapping potential $V_{\text {mag }}$. We will consider systems lying initially in configurations of moderate degeneracy corresponding to $k_{B} T_{i}=0.2-0.5 \epsilon_{F}^{0}$, where $T_{i}$ is the initial temperature 
of the gas. For simplicity, we will assume that also the optical trap can be approximated by a harmonic potential $V_{o p t}$ having a tighter frequency $\omega_{\text {opt }} \gg \omega_{\text {mag }}$, and depth $V_{\text {opt }}(\mathbf{r}=0)=-U$. In our model the trapping potential is then defined as $V_{\text {ext }}(\mathbf{r})=V_{\text {opt }}(\mathbf{r})$ inside the optical trap $\left(V_{\text {opt }}<V_{\text {mag }}\right)$, and $V_{\text {ext }}(\mathbf{r})=V_{\text {mag }}(\mathbf{r})$ outside (see Fig. 1). The maximum number of atoms that can be transferred in the optical trap is given by the value

$$
N_{o p t}=\frac{1}{6}\left(\frac{U}{\hbar \omega_{o p t}}\right)^{3} .
$$

As we will see, if we start from moderately low temperatures Eq.(11) provides an accurate estimate of the number of atoms which are actually transferred by the adiabatic process, provided $N_{\text {opt }} \ll N$. The relative number of atoms transferred in the optical trap is then given by the useful expression

$$
\frac{N_{o p t}}{N}=\left(\frac{U \omega_{m a g}}{\epsilon_{F}^{0} \omega_{o p t}}\right)^{3} .
$$

Typical values that will be considered are $\omega_{\text {mag }} / \omega_{o p t}=$ 0.1 and $U / \epsilon_{F}^{0}=5$, corresponding to $N_{\text {opt }} / N \sim 10 \%$. Since the number of transferred atoms is small one expects that the final temperature $T_{f}$ of the gas will not change significantly with respect to the initial value $T_{i}$. The final degree of degeneracy of the gas will be however significantly higher, since the final Fermi energy is approximately given by $\epsilon_{F} \simeq U+\epsilon_{F}^{0}$. At the same time the central density of the gas, that at small temperature is equal to $n(0)=\left(2 m \epsilon_{F}^{0} / \hbar^{2}\right)^{3 / 2} /\left(6 \pi^{2}\right)$ in the initial stage, will increase by the factor $\left(\epsilon_{F} / \epsilon_{F}^{0}\right)^{3 / 2}$ in the optical trap. The increase of the Fermi energy and of the central density has an important effect on the value of the BCS temperature which is expected to behave, for negative scattering lengths, as [8]

$$
T_{B C S} \sim \frac{\epsilon_{F}}{k_{B}} \exp \left[-\frac{\hbar \pi}{2 p_{F}|a|}\right],
$$

where $p_{F}=\hbar\left(6 \pi^{2}\right)^{1 / 3} n(0)^{1 / 3}$ is the Fermi momentum calculated in the center of the trap. The value of the BCS temperature can increase significantly with respect to its value in the magnetic trap. For example, by taking $a \sim-2000 a_{0}$, a ratio $U / \epsilon_{F}^{0}=5$ and an initial central density $n(0) \sim 10^{12} \mathrm{~cm}^{-3}$, we obtain an increase of the density by a factor $\sim 15$ and the BCS temperature (3) increases by the huge factor $\sim 50$, becoming comparable to the initial value of the Fermi temperature $\epsilon_{F}^{0} / k_{B}$. The above discussion suggests that the proposed adiabatic mechanism might provide conditions of high degeneracy, not far from the transition to the BCS phase. With such a denser gas also the effects of the mean field on the density profile may be significant. An estimate is given by the ratio [7] $E_{\text {int }} / E_{h o} \simeq 0.3 p_{F} a / \hbar$ between the interaction energy and the oscillator energy of a spherically symmetric trap. By using the values employed above one finds corrections of the order of $10 \%$.

The high degeneracy realized in the gas is expected to show up in the velocity distribution and in the release energy. After completing the adiabatic transfer one can release the magnetic trap. Measuring the velocity distribution of these atoms then provides information on the temperature of the system which is expected to be close to the initial value. The atoms of the optical trap can be imaged in a second step. For them one predicts that the ratio $E_{k i n} / k_{B} T_{f} \sim 3 U / 8 k_{B} T_{f}$ between the kinetic energy per particle and the thermal energy, should be enhanced in a significant way if $U \gg k_{B} T_{f}$ revealing the effects of quantum degeneracy.

To confirm the scenario emerging from the above discussion we have carried out a numerical calculation of the thermodynamic functions before and after the adiabatic transformation. The calculation is obtained by imposing that the initial and final configurations have the same entropy. This has been calculated using the semiclassical expression

$$
\begin{aligned}
\frac{S}{k_{B}} & =\frac{1}{(2 \pi \hbar)^{3}} \int d \mathbf{r} d \mathbf{p}\left[\frac{\epsilon(\mathbf{p}, \mathbf{r}) / k_{B} T-\log z}{z^{-1} e^{\epsilon(\mathbf{p}, \mathbf{r}) / k_{B} T}+1}\right. \\
& \left.+\log \left(1+z e^{-\epsilon(\mathbf{p}, \mathbf{r}) / k_{B} T}\right)\right]
\end{aligned}
$$

where $z=\exp \left(\mu / k_{B} T\right)$ is the gas fugacity and

$$
\epsilon(\mathbf{p}, \mathbf{r})=\frac{p^{2}}{2 m}+V_{\text {ext }}(\mathbf{r})
$$

are the semiclassical particle energies. The results are presented in Figs. 2-5. In Fig. 2 we show the relative number of atoms in the optical trap as a function of the depth of the optical trap $U / \epsilon_{F}^{0}$ for two initial temperatures. For the chosen configuration with $\omega_{m a g} / \omega_{o p t}=0.1$ and final depth $U=5 \epsilon_{F}^{0}$, the optical trap can host about $10 \%$ of atoms confirming the analytic prediction (2).

In Fig. 3 the final temperature of the gas is plotted as a function of $U / \epsilon_{F}^{0}$. As already anticipated the final temperature of the gas does not change significantly from the original value, except for values of $U / \epsilon_{F}^{0}$ of the order of $\omega_{\text {opt }} / \omega_{\text {mag }}$. Notice however that, due to the tight confinement, the temperature of the gas can become comparable to the optical oscillator temperature $\hbar \omega_{\text {opt }} / k_{B}$ if $N$ is not large enough.

In Fig. 4 we show the kinetic energy per atom of the gas in units of the final temperature. The initial temperature is $k_{B} T_{i}=0.25 \epsilon_{F}^{0}$. In the same figure, we also show the kinetic energy per particle of the gas occupying separately the magnetic and the optical trap. These values are obtained by averaging separately the kinetic energy over the particles occupying the optical trap $(\epsilon(\mathbf{p}, \mathbf{r})<0)$, and the particles occupying the magnetic $\operatorname{trap}(\epsilon(\mathbf{p}, \mathbf{r})>0)$. The kinetic energy provides an important indicator of the quantum degeneracy of the gas. The 
effect is very spectacular for the atoms of the optical trap, where at $U=5 \epsilon_{F}^{0}$ one finds $E_{k i n} / k_{B} T_{f} \simeq 7$. By choosing a higher initial temperature the effect is less pronounced, but still large $\left(E_{k i n} / k_{B} T_{f} \simeq 3\right.$ for $k_{B} T_{i}=0.5 \epsilon_{F}^{0}$ and $\left.U=5 \epsilon_{F}^{0}\right)$. Because of the high degeneracy the velocity distribution of the atoms in the optical trap deviates significantly from a Boltzmann distribution (Fig. 5). We have also calculated the central density as a function of the depth $U$. For $U=5 \epsilon_{F}^{0}$ and $k_{B} T_{i}=0.25 \epsilon_{F}^{0}$, we find an increase by a factor 15 with respect to the initial value.

In the second part of the work we discuss the conditions needed to achieve the proposed adiabatic transformation. Let us recall that there are several time scales in the problem. A first scale is fixed by the periods of the harmonic wells. These times (of order of $10^{-3}-10^{-1} \mathrm{sec}$ ) are expected to be shorter than the relaxation times due to collisions. The condition of reversibility requires that the relaxation time be much shorter than the time over which the optical trap is switched on. We have simulated the process of thermalization during the gradual increase of the depth of the optical trap by solving the quantum Boltzmann equation. We assume equal distribution functions for the two spin components and that the phasespace distribution of particles is a function only of the single-particle energies $\epsilon(\mathbf{p}, \mathbf{r})$ (ergodic assumption) 6, 2, 2. This yields the following equation to solve

$$
\begin{aligned}
\rho\left(\epsilon_{1}\right) \frac{\partial f\left(\epsilon_{1}\right)}{\partial t} & =\frac{m \sigma}{\pi^{2} \hbar^{3}} \int d \epsilon_{3} d \epsilon_{4} \rho\left(\epsilon_{\text {min }}\right) \\
& \times\left[f\left(\epsilon_{3}\right) f\left(\epsilon_{4}\right)\left(1-f\left(\epsilon_{1}\right)\right)\left(1-f\left(\epsilon_{2}\right)\right)\right. \\
& \left.-f\left(\epsilon_{1}\right) f\left(\epsilon_{2}\right)\left(1-f\left(\epsilon_{3}\right)\right)\left(1-f\left(\epsilon_{4}\right)\right)\right],
\end{aligned}
$$

where $\rho(\epsilon)$ is the density of states in the potential $V_{e x t}$, which we model by $\rho(\epsilon)=(\epsilon+U)^{2} / 2\left(\hbar \omega_{\text {opt }}\right)^{3}$ if $\epsilon<$ 0 , and $\rho(\epsilon)=\epsilon^{2} / 2\left(\hbar \omega_{\text {mag }}\right)^{3}$ if $\epsilon>0$. Furthermore, $\epsilon_{\min }=\min \left\{\epsilon_{1}, \epsilon_{2}, \epsilon_{3}, \epsilon_{4}\right\}$ is the minimum value of the four single-particle energies involved in the collisions and $\epsilon_{2}=\epsilon_{3}+\epsilon_{4}-\epsilon_{1}$ according to energy conservation. The cross-section for collisions between the two distinguishable spin states is $\sigma=4 \pi a^{2}$ and is fixed by the $s$-wave scattering length $a$. In the numerical simulation the depth $U$ of the optical trap is ramped up by keeping the ratio $\omega_{m a g} / \omega_{\text {opt }}$ constant, and at each step we let the gas thermalize in the new configuration. From Eq. (6), it turns out that the time scale of thermalization is fixed by $\tau^{-1}=\omega_{m a g}\left(|a| / a_{m a g}\right)^{2} N^{2 / 3}$, where $a_{m a g}=$ $\left(\hbar / m \omega_{\text {mag }}\right)^{1 / 2}$ is the magnetic oscillator length. If we choose $\omega_{\text {mag }} / \omega_{\text {opt }}=0.1, U=5 \epsilon_{F}^{0}$ and $k_{B} T_{i}=0.5 \epsilon_{F}^{0}$, we find that the time required for the reversible transformation is $t_{\text {rev }} \simeq 100 \tau$. For a configuration with $N=10^{6}$, $\omega_{\text {mag }}=2 \pi \times 100 \mathrm{~Hz}$ and $|a| / a_{\text {mag }}=5 \times 10^{-3}$, this corresponds to $t_{\text {rev }} \sim 1 \mathrm{sec}$. If one starts from the lower initial temperature $k_{B} T_{i}=0.25 \epsilon_{F}^{0}$ and keeps the other parameters unchanged, the time $t_{r e v}$ turns out to be about a factor two shorter. The origin of this behavior is due to the mechanism of atom exchange between the magnetic and the optical trap. Notice, however, that $t_{\text {rev }}$ depends rather strongly on the value of $N$ and becomes longer if $N$ decreases.

The true shape of the optical trap differs from the harmonic potential employed above. Differences appear because a dipole trap can be safely approximated by a harmonic potential only at its center and, in general, can host more atoms than the corresponding harmonic trap with the same depth $U$ and frequency ratio $\omega_{m a g} / \omega_{\text {opt }}$. This results in more heating and, as a consequence, in a higher final temperature $T_{f}$. For $k_{B} T_{i}=0.25 \epsilon_{F}^{0}$, we find that the fraction of atoms in the optical trap at $U=5 \epsilon_{F}^{0}$ is about $50 \%$ larger than the value obtained using the harmonic approximation, while the final temperature is only about $10 \%$ higher. The final degree of degeneracy of the gas in the optical trap is consequently slightly reduced, but the qualitative features discussed above remain unchanged.

If the time of the transformation is longer than the inverse oscillator frequency, but faster than the relaxation time (a relatively easy condition to realize experimentally), then the transformation will be adiabatic, in the sense of entropy conservation, but the system will not be in thermal equilibrium. Such a transformation corresponds to an adiabatic transfer of the lowest $N_{\text {opt }}$ single particle states from the magnetic to the optical trap. The transformation keeps the corresponding occupation numbers unchanged. In this case, the atoms in the optical trap form a cold, out of equilibrium gas. With the initial condition $k_{B} T_{i}=0.5 \epsilon_{F}^{0}$ this out-of-equilibrium transformation transfers approximately the same fraction of atoms into the optical trap as with the fully reversible transformation discussed above and the average kinetic energy of these atoms takes a similar value, revealing that the system is highly degenerate. By using the quantum Boltzmann equation (6) we have found that the time needed for this configuration to relax to equilibrium is significantly shorter, the final temperature of the gas being only sligthly higher than the one obtained in the reversible transformation.

In conclusion we have investigated the consequences of an adiabatic transfer of a gas of Fermions from a magnetic to a tighter optical trap. We have seen that it is possible to reach configurations involving a significant fraction of atoms which, as a result of the transformation, will occupy a Fermi sea in conditions of high degeneracy. The large enhancement of the release energy should be easily observable through time of flight measurements. This method could make it possible to produce highly degenerate Fermi gases, which, in the case of gases interacting with negative scattering length, are close to the transition to the BCS phase.

Useful discussions with Brian DeMarco are acknowledged. It is also a pleasure to thank Wolfgang Ketterle for useful comments. L. V. wishes to thank the Danish Ministry of Education for support. This research was 
supported by Ministero dell'Università e della Ricerca Scientifica e Tecnologica (MURST).

[1] B. DeMarco and D.S. Jin, Science 285, 1703 (1999).

[2] M.J. Holland, B. DeMarco and D.S. Jin, Phys. Rev. A 61 053610 (2000).

[3] M. Crescimanno, C.G. Kaoy and R. Peterson, Phys. Rev. A 61053602 (2000); W. Geist, A. Idrizbegovic, M. Marinescu, T.A.B. Kennedy and L. You, Phys. Rev. A 61 013406 (2000).

[4] D.M. Stamper-Kurn, H.-J. Miesner, A.P. Chikkatur, S. Inouye, J. Stenger and W. Ketterle, Phys. Rev. Lett. 81, 2194 (1998).

[5] P.W.H. Pinkse, A. Mosk, M. Weidemüller, M.W. Reynolds, T.W. Hijmans and J.T.M. Walraven, Phys. Rev. Lett. 78, 990 (1997).

[6] O.J. Luiten, M.W. Reynolds and J.T.M. Walraven, Phys. Rev. A 53, 381 (1996).

[7] L. Vichi and S. Stringari, Phys. Rev. A 60, 4734 (1999).

[8] H.T.C. Stoof and M. Houbiers in Bose-Einstein Condensation in Atomic Gases, Proceedings of the International School of Physics "Enrico Fermi" edited by M. Inguscio, S. Stringari and C.E. Wieman (SIF, Bologna, 1999).

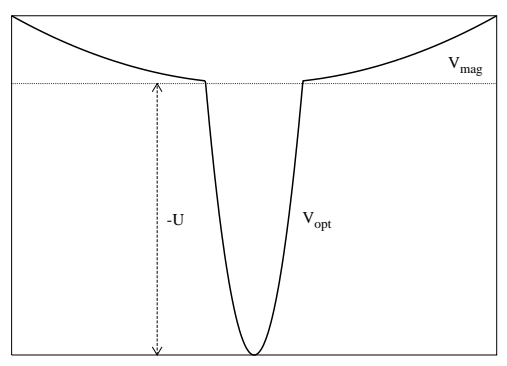

FIG. 1. Schematic representation of the confining potential $V_{\text {ext }}(\mathbf{r})$ in the presence of the magnetic and optical traps.

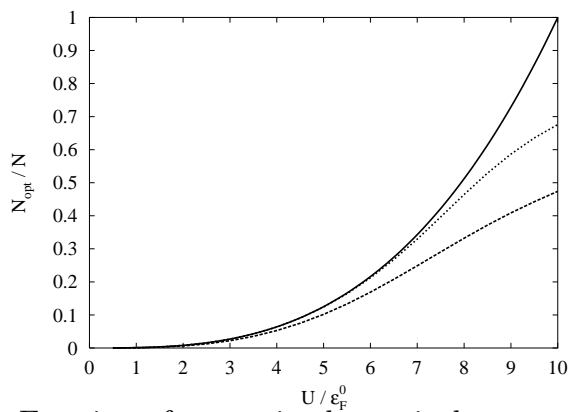

FIG. 2. Fraction of atoms in the optical trap as a function of $U / \epsilon_{F}^{0}$ for two initial temperatures: $k_{B} T_{i}=0.5 \epsilon_{F}^{0}$ (dashed line), and $k_{B} T_{i}=0.25 \epsilon_{F}^{0}$ (dotted line). The solid line corresponds to the analytical estimate (2).

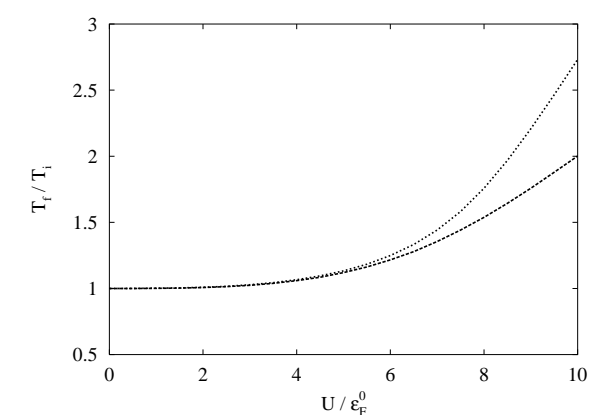

FIG. 3. Ratio between the final and initial temperature of the gas as a function of $U / \epsilon_{F}^{0}$ for the same initial temperatures as in Fig. 2.

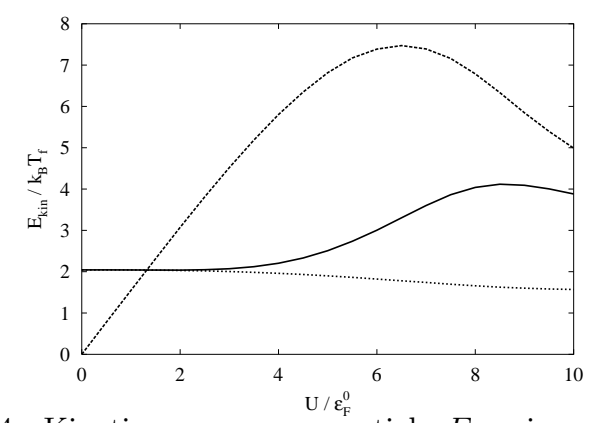

FIG. 4. Kinetic energy per particle $E_{k i n}$ in units of the final temperature $k_{B} T_{f}$ as a function of $U / \epsilon_{F}^{0}$. The initial temperature is $k_{B} T_{i}=0.25 \epsilon_{F}^{0}$. The solid line corresponds to the kinetic energy per particle averaged over the entire system. Also shown are the kinetic energy per particle in the optical trap (dashed line), and in the magnetic trap (dotted line).

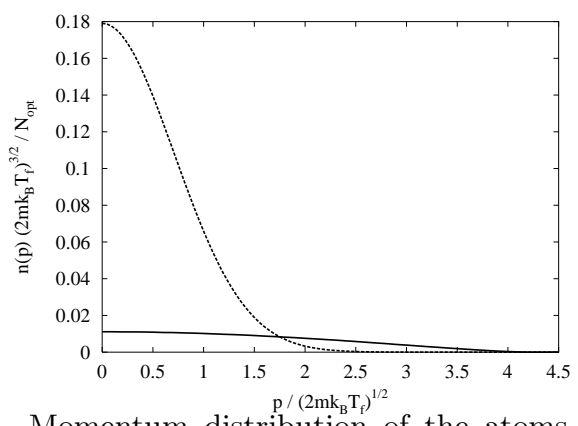

FIG. 5. Momentum distribution of the atoms occupying the optical trap with $U=5 \epsilon_{F}^{0}$ (solid line). The initial value of the temperature is $k_{B} T_{i}=0.25 \epsilon_{F}^{0}$. The dashed line corresponds to a Boltzmann gas of the same number of atoms at the temperature $T=T_{f}$. 